\title{
Comprehensive verticality analysis and web- based rehabilitation system for people with multiple sclerosis with supervised medical monitoring
}

\author{
Gonzalo Eguiluz-Perez * and Begonya Garcia-Zapirain \\ DeustoTech-Life Unit, DeustoTech Institute of Technology, University of Deusto, Bilbao 48007, \\ Spain
}

\begin{abstract}
People with Multiple Sclerosis (MS) need regular physical activities along with medical treatment despite their ability or disability level. However, poorly performed exercises could aggravate muscle imbalances and worsen their health. The goal of our work is to create a comprehensive system, encompassing a face-to-face sessions performed by MS patients one day a week at the medical center with exercises at home the rest of the week through a web platform in combination with a tracking tool to analyze the position of patients during exercise and correct them in real-time. The whole system is currently testing during six months with ten participants, five persons with MS and 5 professionals related with MS. Two tests, the Functional Independence Measure and the Berg Balance Scale will be act as a barometer for measuring the degree of independence obtained by the people with MS and also the validity of the whole system as a rehabilitation tool. Preliminary results about the usability of the system using SUS scale, 72 and 76 points over 100 (patients and professionals respectively), demonstrate that our system is usable for both patients and professionals.
\end{abstract}

Keywords: Multiple Sclerosis, telerehabilitation, web application, verticality analysis

\section{Introduction}

Multiple sclerosis (MS) is a chronic illness which affects brain and spinal cord that causes a handicap for the affected person both physically and emotionally [1]. Scientist have not discovered a cure for MS yet, although regular exercise combined with special chemicals can help patients manage symptoms and improve their health condition. Moreover, no two patients are alike in their circumstances or their symptoms, so two treatments cannot be equal. The focus of this paper will be on the physical part of the treatment and the tools designed for a better management of MS disease.

The physical treatment has to carry out correctly. Poorly carried out exercises could aggravate muscular problems which also contributes to the worsening of physical health. The way patients sit, stand

\footnotetext{
${ }^{*}$ Corresponding author: Gonzalo Eguiluz-Perez, DeustoTech-Life Unit, DeustoTech Institute of Technology, University of Deusto, Bilbao 48007, Spain. E-mail: geguiluz@deusto.es.
} 
and walk has a long-term effect on the musculoskeletal system [2,3]. The objectives that this project aims to achieve are the following:

- To incorporate treatment techniques in livelihoods through a web application, relating them with appropriate daily activities, providing this way maintenance of all the improvement obtained.

- To stimulate any sensitive and perceptual experience, and maintain the experience of the normal movement throughout the progression of the disease.

- To perform real time analysis of patients' position, verticality of the trunk, during workout session to avoid unsatisfactory practices that may result in severe muscle injuries.

The paper is organized as follows: first of all, the materials and methods section gives details of the experiment. System design section describes the technological solution and the findings of the study are then presented in experiment results section, followed by a conclusion explanation.

\section{Previous work}

Telerehabilitation is the provision of rehabilitation services at a distance using telecommunications technology. Telerehabilitation systems provide a continuity of care with potential time and costsavings. Examples of these systems are described below [4-6]:

- eRehab (ubiquitous multi-device personalized telerehabilitation platform) [7]. This project aims to develop and validate the eRehab platform, a tele-rehabilitation platform based on personalized health service massive deployment architecture to carry out therapies in different environments.

- CLEAR (Clinical Leading Environment for the Assessment and validation of Rehabilitation Protocols for home care) Project [8]. Under this project a large scale Pilot study has been conducted in four Member States of the European Union (Italy, Spain, The Netherlands and Poland) to demonstrate the feasibility of a Tele-rehabilitation service using Habilis, a general-purpose software platform.

- Tereha [9]. Tereha is an integrated platform covering motor, cognitive and psychological rehabilitation for patients with brain damage through the use of ICT Technologies and Natural Interfaces. The platform consists of three modules: physical, cognitive and psychological rehabilitation. The first two use virtual rehabilitation systems (augmented reality), based on simple games that will help improve the patients' skills and abilities.

- Ubiquitous Cardiac Care (UCC) [10]. The UCC system adopts pervasive service and IT technology, and infuses pervasive computing concept into healthcare services. UCC is a new attempt of IoT (Internet of Things) technology in the field of health care, i.e. smart care.

\section{Materials and methods}

\subsection{Materials}

EM (Multiple Sclerosis Basque Foundation) professionals have created a repository of exercises to populate the web system. The repository consists of pictures in popular formats (JPG, PNG and GIF), videos in Flash format (FLV) and documents in Microsoft Office format (DOC), which are categorized into six main categories (with 192 subcategories): Rehabilitation, Physiotherapy, Speech Therapy, Occupational Therapy, Neuropsychology and Yoga. Table 1 shows the distribution of the multimedia material over the categories. 
Table 1

Multimedia distribution over main categories

\begin{tabular}{llll}
\hline Category & Pictures & Videos & Documents \\
\hline Rehabilitation & $\mathrm{x}$ & $\mathrm{x}$ & \\
\hline Physiotherapy & $\mathrm{x}$ & $\mathrm{x}$ & $\mathrm{x}$ \\
\hline Speech Therapy & $\mathrm{x}$ & $\mathrm{x}$ & \\
\hline Occupational Therapy & $\mathrm{x}$ & & $\mathrm{x}$ \\
\hline Neuropsychology & & & $\mathrm{x}$ \\
\hline Yoga & & $\mathrm{x}$ & \\
\hline & & \\
\hline Number of elements & 307 & 201 & 140 \\
\hline Size & $162 \mathrm{MB}$ & $1.45 \mathrm{~GB}$ & $21 \mathrm{MB}$ \\
\hline
\end{tabular}
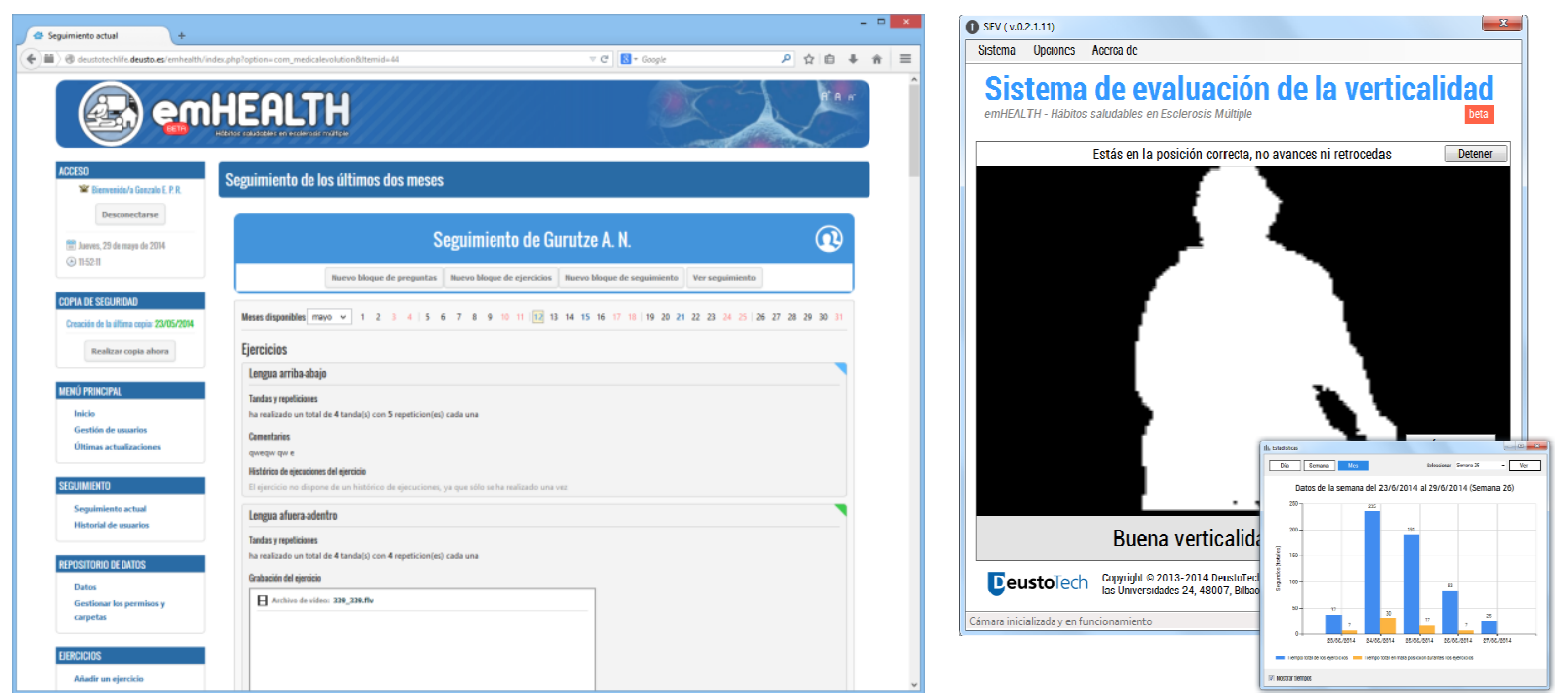

Fig. 1. Web application (left) and ad-hoc desktop application (right).

Along with this, a telerehabilitation web-based system was developed to offer another way to carry out the rehabilitation, combining traditional rehabilitation at the medical center with sessions at patient's home. Also, an ad-hoc tracking system is being developed to track the posture of patients during the rehabilitation session. Both web system and tracking system will be explained in detail in section 4 system design. Figure 1 shows the web application with the professional's view and the ad-hoc desktop application with a patient using the system.

\subsection{Participants}

To obtain the sample, a group of 20 people belonging to EM were invited to participate, differentiating between two groups: medical professionals and people with multiple sclerosis. For none of two groups no distinctions were made in recruitment on grounds of origin, ethnicity, religious belief or social status. However, for the group of people with MS, different inclusion and exclusion criteria were defined:

- Collective of people with MS: The requirements to participate in the pilot were: (1) Must be 18 to 56 years of age, inclusive, (2) have a diagnosis of multiple sclerosis, (3) must have a baseline 
EDSS between 0.0 and 4.0, inclusive and (4) must be able to use a PC, a web browser and a desktop application. Furthermore, the exclusion criteria comprised $(1)<18$ years of age or $>=56$ years of age, (2) EDSS score $>4.0$, and (3) a severe cognitive impairment (MMSE $<24$ ).

For that reason, only $33 \%$ of candidates with MS, 5 out of $15(n=5)$, were eligible for the test period, 3 women and 2 men. Moreover, in the medical group, five professionals volunteered to test the platform, 4 women and 1 man. In short, 10 persons are carrying out the test.

\subsection{Methods}

A professional met patients individually at EM facilities in one session that lasted 30 minutes on average to explain the aim of the pilot test and its duration. Next day, both professional and patient had another session, 45 minutes on average, to explain everything about the whole system (web system and desktop application). The system was installed in participants' home, a PC and a DS311 camera connected to Internet by one research member. During three weeks, patients tested the system to look for problems and deal with them. Following initial contact, for 20 weeks, professionals combined visits to the medical center with assigned exercises through the web platform. In this way, patients performed these exercises via Internet at home using the tracking system at the same time, with a minimum degree of supervision.

Before the initial of the test period, professionals passed two tests to participants involved in the pilot, the Functional Independence Measure (FIM) [11] and the Berg Balance Scale (BBS) [12]. FIM provides a uniform system of measurement for disability based on the International Classification of Impairment, Disabilities and Handicaps. It measures the level of a patient's disability and indicates how much assistance is required for the individual to carry out activities of daily living. BBS, in turn, is a 14-item objective measure designed to assess static balance and fall risk in adult populations which can be used with other collectives like MS.

After the first four weeks of testing, both professionals and patients filled a satisfaction survey focused on the usability of the system. This satisfaction survey was based on a 10 item questionnaire with 5 response options, with values from 0 to 4; a System Usability Scale (SUS) [13] questionnaire.

The test is currently under way and on pilot completion, both the FIM and the BBS tests will be passed again to measure the evolution of patients and to evaluate if the system offers successful results.

\section{System design}

In one hand, the web application is modular-designed, and each feature is represented by a module. Joomla, a Content Management System (CMS), acts as the infrastructure for data and modules handling. The data storage is powered by MySQL. Finally, the system runs in a Linux environment, in particular, Ubuntu Server environment, with Apache server.

To create these modules, WEB technologies like $\mathrm{PHP}^{1}$, JavaScript ${ }^{2}$ and $\mathrm{AJAX}^{3}$ have been used, along with multimedia technologies like Red $5^{4}$ and Adobe Flex ${ }^{5}$.The choice of Adobe Flex instead of

\footnotetext{
${ }^{1} \mathrm{PHP}$ is a widely-used Open Source general-purpose scripting language that is especially suited for web development.

${ }^{2}$ JavaScript is a dynamic scripting language supporting prototype based object construction.

${ }^{3} \mathrm{AJAX}$ is a web development technique for creating RIAs running on the client's browser, maintaining communication asynchronously with the server in the background.

${ }^{4}$ RED5 is an Open Source server for presenting stream contents in Adobe Flash using RTMP/RTMPT/RTMPS.
} 


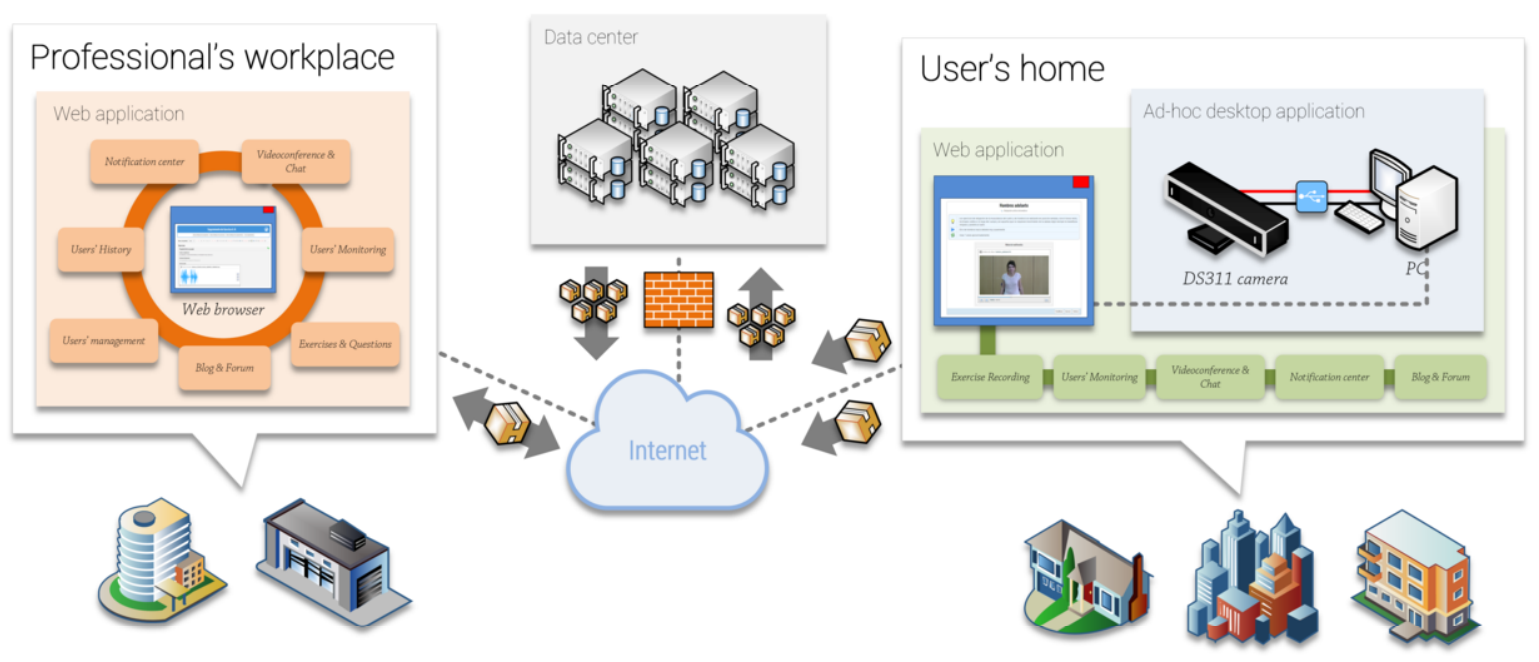

Fig. 2. High level diagram.

HTML5 is because HTML5 is still a draft and HTML5's necessary APIs for webcam access, getUserMedia API, can't run properly on Internet Explorer, Safari and older versions of Google Chrome, Mozilla Firefox and Opera.

The System has two views, (see Figure 2), one for the doctors/physiotherapist (in short professionals) and one for the patients:

- Professional's view contains everything necessary to ensure efficient monitoring as well as all the necessary to provide content to the system. To add content, the professionals have several modules: exercises, questions, repository, forum, blog and users' management. To monitor users, the professionals have the monitoring module and the history module, which acts like the monitoring module but with the ability to select dates. Finally, professional view has a chat, videoconference and a notification center module.

- Patients' view offers a personalized monitoring module and forum, blog, videoconference, chat, recording and notification center modules.

Figure 3 shows the main modules of the system. As stated above, the system is designed on the basis of modularity, and each module is independent. Every module is described below:

- Exercise recording module (1). This module is exclusive to patients; by means of it, every patient has the possibility to record the exercise during the execution.

- Users' management module (2). This module is used for creating users and for controlling system and account policies.

- Exercises and Questions modules (3). Only the professionals are able to use these modules. With these modules, the professionals can set up a repository of exercises and questions.

- Users' History module (4). History module acts like the monitoring module but whit the option to choose between two dates.

- Blog and Forum modules (5). Forum and blog offer offline communication. These modules make it possible to create thematic threads involving both professionals and patients.

- Notification center (6). This module shows the latest updates in the system.

\footnotetext{
${ }^{5}$ Adobe Flex Flex allows programmers to rapidly develop cross-platform applications and their layouts using MXML and ActionScript language.
} 


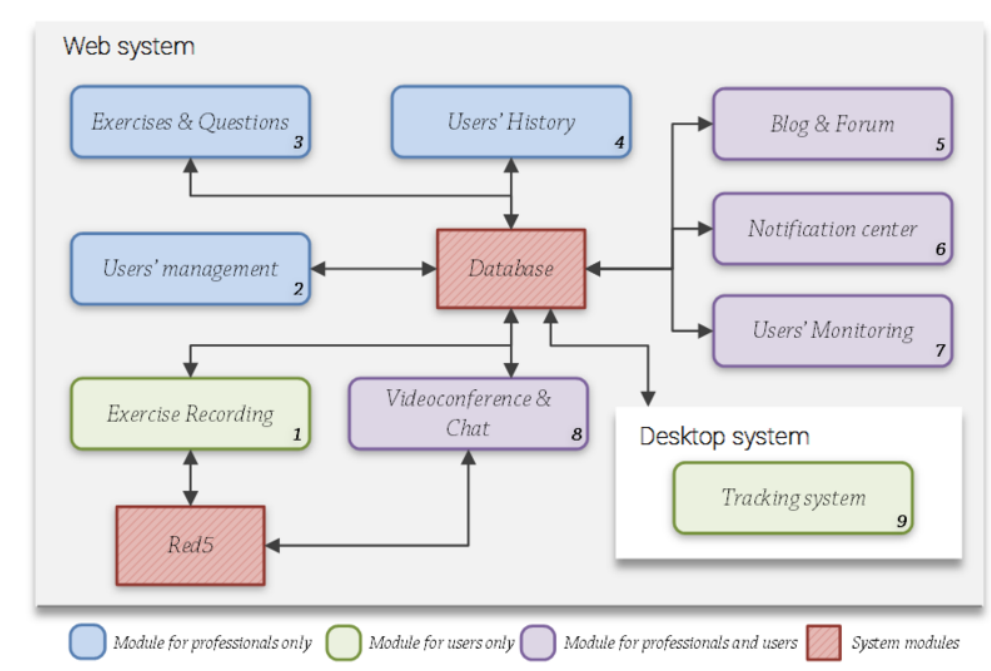

Fig. 3. Web and desktop systems' block diagram.

- Users' Monitoring module (7). Monitoring module allows the professional to see the evolution of each patients, day-by-day, and enables a personalized treatment, based on the characteristics and the evolution of the patient.

- Chat and Videoconference modules (8). Both use Red5 technology. Those modules offer a direct link between the professional and the patient, either by text or by video/audio.

On the other hand, the ad-hoc desktop application (9) uses image processing algorithms to detect the position of the person, in particular, the verticality of the patient's trunk (positions leaning forward or leaning backwards not included), and taking advantage of depths images provided by the camera sensors. In order to achieve this, a Time-of-Flight (ToF) camera has been used, specifically the Depthsense ${ }^{\circledR} 311$ (DS311) camera developed by SoftKinetic, Inc. A ToF camera works by illuminating the scene with a modulated light source, and observing the reflected light. The phase shift between the illumination and the reflection is measured and translated to distance. The tracking system consists of three main blocks: Acquire data, RGB and Depth Data Processing Algorithm and Correction algorithm. The first block is executed by the camera automatically, and both the RBG and the depth data acquisition is done simultaneously by the camera through two lenses, one for acquiring color images and one for acquiring depth images. The image processing algorithm is based on two steps executed consecutively. The first one is the background subtraction and silhouette generator. To achieve this, a Joint Bilateral Filter is used, replacing the intensity value at each pixel by a weighted average of intensity values from nearby pixels, combining RGB and depth data. The next step is attaching geometric shapes. Once the silhouette is calculated, we approximate the silhouette to regular shapes like circles or ovals. To improve the recognition, fuzzy logic is used to associate degrees of certainty to recognized shapes. Two member functions were defined for size and shape to be used in the fuzzy system. The final step, the correction system, calculates the centroids of the shapes generated in the previous step and calculates the angle between those points to inform the user if he or she is in a bad/good posture. At the same time, the system collects the total exercise time and time in bad position, so the data would be used over time to assess the evolution of every patient. 
Table 2

Initial FIM and BBS data

\begin{tabular}{lll}
\hline Patient & Initial FIM (out of 126 points) & Initial BBS (out of 56 points) \\
\hline$\# 1$ & 59 & 39 \\
\hline$\# 2$ & 62 & 40 \\
\hline$\# 3$ & 85 & 46 \\
\hline$\# 4$ & 98 & 50 \\
\hline$\# 5$ & 102 & 52 \\
\hline
\end{tabular}

Table 3

SUS questionnaire and responses

\begin{tabular}{|c|c|c|c|c|c|c|c|c|c|c|c|}
\hline \multirow{2}{*}{ \# } & \multirow{2}{*}{ Questions } & \multicolumn{5}{|c|}{ Professionals } & \multicolumn{5}{|c|}{ Users } \\
\hline & & $\bar{M}$ & $\mathrm{~F}$ & & & & $\mathrm{M}$ & & $\mathrm{F}$ & & \\
\hline 1 & I think that I would like to use this system frequently & 3 & 3 & 4 & 3 & 4 & 3 & 3 & 3 & 4 & 2 \\
\hline 2 & I found the system unnecessarily complex & 1 & 1 & 2 & 2 & 0 & 2 & 2 & 1 & 2 & 1 \\
\hline 3 & I thought the system was easy to use & 3 & 3 & 2 & 2 & 3 & 4 & 3 & 3 & 4 & 3 \\
\hline 4 & $\begin{array}{l}\text { I think that I would need the support of a technical person to be able to use this } \\
\text { system }\end{array}$ & 0 & 1 & 1 & 1 & 0 & 2 & 1 & 2 & 2 & 1 \\
\hline 5 & I found the various functions in this system were well integrated & 3 & 3 & 4 & 3 & 3 & 1 & 4 & 3 & 3 & 3 \\
\hline 6 & I thought there was too much inconsistency in this system & 1 & 2 & 1 & 2 & 1 & 2 & 1 & 1 & 2 & 1 \\
\hline 7 & I would imagine that most people would learn to use this system very quickly & 4 & 3 & 3 & 4 & 3 & 3 & 3 & 3 & 3 & 4 \\
\hline 8 & I found the system very cumbersome to use & 1 & 2 & 1 & 1 & 0 & 2 & 2 & 1 & 0 & 2 \\
\hline 9 & I felt very confident using the system & 3 & 3 & 4 & 4 & 3 & 3 & 3 & 4 & 3 & \\
\hline 10 & I needed to learn a lot of things before I could get going & 1 & 2 & 2 & 1 & 1 & 2 & 2 & 1 & & \\
\hline
\end{tabular}

\section{Results}

Table 2 shows the initial FIM and BBS values of patients before the pilot test. This information may help to compare the obtained data before and after the test and decide if the proposed solution and approach is valid or not when the pilot test ends.

Table 3 shows the SUS (System Usability Scale) questionnaire, together with the responses of both professionals and patients. SUS questionnaire is a simple ten-item attitude Likert scale giving a global view of subjective assessments of usability. Likert scales are commonly used to measure attitude, providing a range of responses to a given question or statement. Typically, there are 5 categories of response, from 0 -strongly disagree to 4-strongly agree. Odd questions are added to total points and even questions are deducted from 4 and then added to total points. Finally, total value is multiply by 2.5 to obtain a value over 100 . This questionnaire has been used to evaluate the usability of the web-based rehabilitation system.

Quantitative variables were measured using descriptive statistics (mean \pm standard deviation). With respect to patients, they considered the system adequate in terms of usability and ease of use (72 \pm 1.4577). In this case, the developed system came in $2^{\text {nd }}$ quartile, achieving the acceptable value in terms of acceptability ranges (not acceptable less than 50 points, marginal between 51 and 70 points and acceptable, more than 70 points).

On the other hand, professionals also considered the system adequate in terms of usability and ease of use $(76 \pm 3.0207)$. In this case, the developed system came in $3^{\text {rd }}$ quartile, achieving the acceptable value in terms of acceptability ranges. 


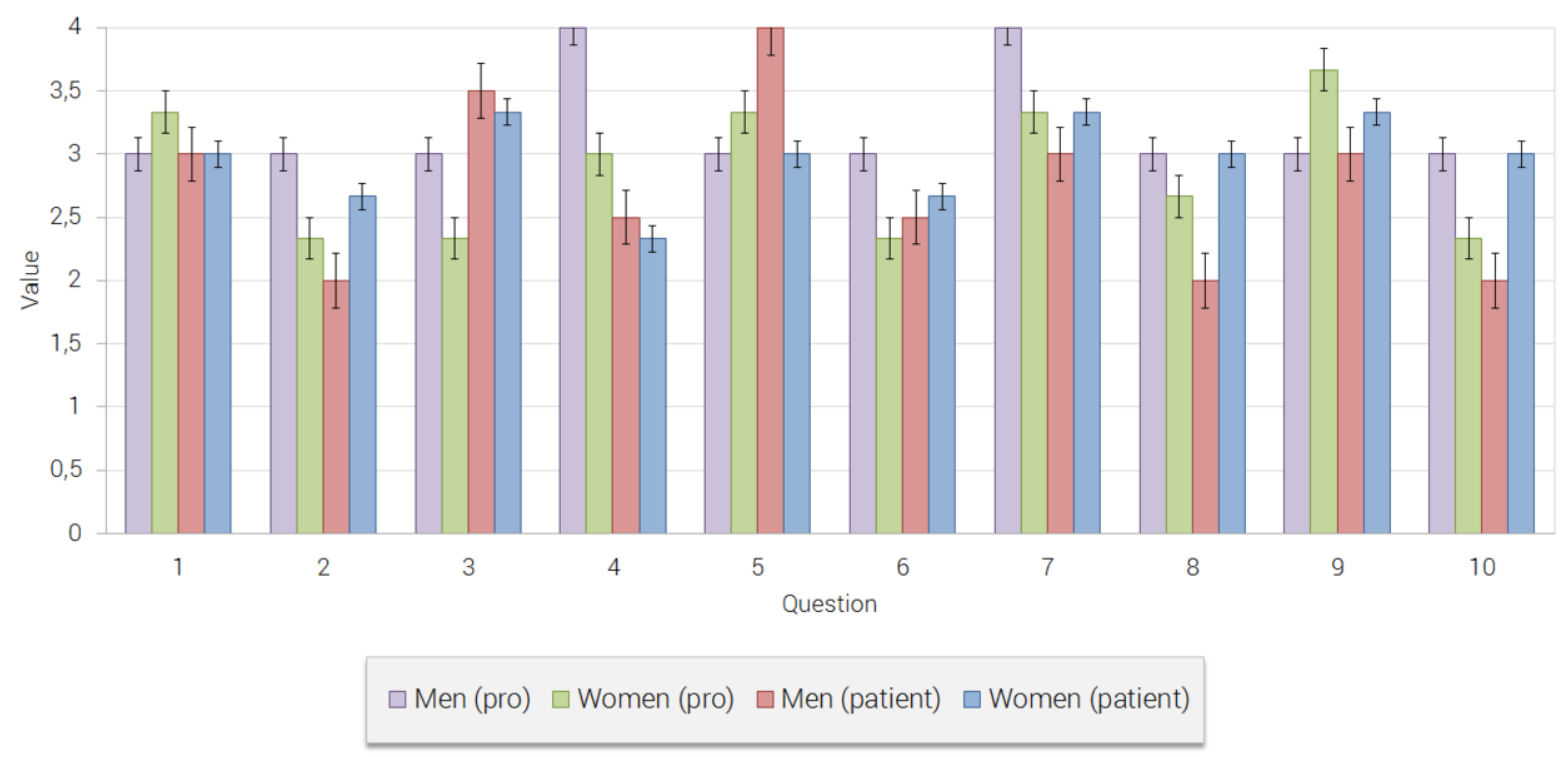

Fig. 4. Questions' average values.

Table 4

MS systems' comparison

\begin{tabular}{lllll}
\hline System & Target & System architecture & Remote & Business model \\
\hline REOVIEM [14] & $\begin{array}{l}\text { - MS } \\
\text { - Rehabilitation }\end{array}$ & $\begin{array}{l}\text { - Software (Desktop application) } \\
\text { - Hardware (Microsoft Kinect) }\end{array}$ & No & No \\
\hline VirtualRehab [15] & MS & $\begin{array}{l}\text { - Software (Desktop application) } \\
\text { - Cloud storage (Microsoft Azure) } \\
\text { - Hardware (Microsoft Kinect) }\end{array}$ & Unlimited SaaS & $\begin{array}{l}\text { - Basic OnPremises } \\
\text { - Unlimited OnPremises } \\
\text { - Unlimited SaaS }\end{array}$ \\
\hline \multirow{2}{*}{ HBPT [16] } & MS & $\begin{array}{l}\text { - HAT units } \\
\text { - HAT server }\end{array}$ & \multirow{2}{*}{ Yes } \\
\hline Our system & - MS & $\begin{array}{l}\text { - Software (Web application) } \\
\text { - Hardware (SoftKinetic DS311) }\end{array}$ & Yes & No \\
\hline
\end{tabular}

\section{Conclusion}

The following table shows a comparison between the developed system and some systems related with MS. All of them offer a hardware-software solution, focusing on physical rehabilitation, but not all offer telerehabilitation. VirtualRehab is a paid system which is available in three versions (see Table 3). All three versions are fee-paying, and the basic version only supports 25 patients. The developed system offers not only physical rehabilitation, but also rehabilitation in other areas of interest as Physiotherapy, Occupational Therapy, etc. Also, the developed system can be adapted easily to other rehabilitation processes, without mentioning that web-based development offers access to the system to a large number of devices like smartphones, tablets, etc.

As stated previously in this manuscript (section 5), the users agreed that the system is usable and easy to use. Technology is getting more and more common in daily routine, and this opens up new possibilities for systems like the proposed. The ease of use is one of the advantages of this system, and the users' feedbacks prove us right. Altogether, the two systems offer a comprehensive telerehabilita- 
tion system covering the main incidence areas of MS and facilitate the work of professionals and patients' rehabilitation.

The web-based system offers, in one hand, a new way for MS patients to continue with their treatments outside the medical center, and, in the other hand, a tool to improve the management of MS disease by professionals. Carrying out the rehabilitation at home is a great step forward in users' quality of life, because they don't need to travel, they don't need to wait for treatment, they can decide when and where they want to carry out the rehabilitation, etc., emphasizing the patient's empowerment. It's also an improvement for medical professionals because they can provide a personalized treatment for several patients at the same time, maintaining the quality of service reaching more people. Other important advantage of this system is the reduction of costs. This is the result of, among other things, the reduction of trips undertaken by users and of the low-budget infrastructure needed to host the whole system.

For its part, the verticality analysis tool offers effective support to patients when carrying out the exercises at home. The lack of professional supervision is replaced with the developed tool. Thus, the patient feels more secure and confident wanting to continue with their rehabilitation at home. This is also beneficial for professionals because the tool ensures a correct level of the realization of physical rehabilitation exercises. The application of time-of-flight technology is novel in the area, and only the Kinect (the technology used in Kinect is very similar to structured light ${ }^{6}$ and it is called light coding [17]) sensor has been used in similar systems as seen before in Table 3.

After this point, the way forward is to include more areas of analysis like joints, which is vital in quantifying numerically the rehabilitation process. An automatic process which analyzes the elements involved in an elbow motion or a knee motion, for example, would be a significant step forward in patient empowerment, which is the key for near future health systems.

In summary, the goal of this project was and is to provide, with ultimate flexibility and ease of use, a new way to manage MS disease, for both medical professionals and patients, using the technologies that surround us; and the information collected through the SUS questionnaires indicates that we are going in the right direction.

\section{Acknowledgment}

We would like to express our gratitude to BIZKAILAB initiative of the Biscay Council, EM and the Basque Country Department of Education, Universities and Research for their support. Also, we wish to acknowledge the University of Deusto, which kindly lent infrastructures and materials.

\section{References}

[1] G. Eguiluz-Perez and B. Garcia-Zapirain, Use of a time-of-flight camera with an omek beckon ${ }^{\mathrm{TM}}$ framework to analyze, evaluate and correct in real time the verticality of multiple sclerosis patients during exercise, International Journal of Environmental Research and Public Health 11 (2013), 5807-5829.

[2] K. Fowler and L. Kravitz, The perils of poor posture, Idea Fitness Journal 8 (2011).

[3] C.H. Yu, S.H. Shin, H.C. Jeong, D.Y. Go and T.K. Kwon, Activity analysis of trunk and leg muscles during whole body tilt exercise, Bio-Medical Materials and Engineering 24 (2014), 245-254.

[4] D. Hailey, R. Roine, A. Ohinmaa and L. Dennett, Evidence of benefit from telerehabilitation in routine care: A systematic review, J. Telemed. Telecare. 17 (2011), 281-287.

\footnotetext{
${ }^{6}$ Structured light is the projection of a light pattern (plane, grid, or more complex shape) at a known angle onto an object.
} 
[5] D. Hailey, A. Ohinmaa and R. Roine, Study quality and evidence of benefit in recent assessments of telemedicine, J. Telemed. Telecare 10 (2004), 318-324.

[6] E. Whitworth, J.A. Lewis, R. Boian, M. Tremaine, G. Burdea and J.E. Deutsch, Formative evaluation of a virtual reality telerehabilitation system for the lower extremity, Proceedings of the 2nd International Workshop on Virtual Rehabilitation (IWVR2003), Piscataway, NJ, USA (2003), 21-22.

[7] G. Epelde, E. Carrasco, I. Gomez-Fraga, K. Vivanco, J.M. Jimenez, O. Rueda, A. Bizkarguenaga, D. Sevilla and P. Sanchez, ERehab: Ubiquitous multidevice personalised telerehabilitation platform, Proceedings of the AAL Forum 2012, Eindhoven, Netherlands (2012), 24-27.

[8] M. Zampolini, E. Todeschini, G.M. Bernabeu, H. Hermens, S. Ilsbroukx, V. Macellari and C. Giacomozzi, Telerehabilitation: Present and future, Ann. Ist Super Sanita 44 (2007), 125-134.

[9] R. Lloréns, C. Colomer-Font, M. Alcañiz and E. Noé-Sebastián, BioTrak virtual reality system: Effectiveness and satisfaction analysis for balance rehabilitation in patients with brain injury, Neurologia (English Edition) 28 (2013), 268275.

[10] J. Li, H. Zhou, D. Zuo, K.M. Hou and C. De Vaulx, Ubiquitous health monitoring and real-time cardiac arrhythmias detection: a case study, Bio-Medical Materials and Engineering 24 (2014), 1027-1033.

[11] C.V. Granger et al., Advances in functional assessment for medical rehabilitation, Topics in Geriatric Rehabilitation 1 (1986), 59-74.

[12] K. Berg, S. Wood-Dauphinee and J.I. Williams, The balance scale: Reliability assessment with elderly residents and patients with an acute stroke, Scandinavian Journal of Rehabilitation Medicine 27 (1995), 27-36.

[13] A. Bangor, P. Kortum and J. Miller, Determining what individual SUS scores mean: Adding an adjective rating scale, J. Usability Stud. 4 (2009), 114-123.

[14] J.A. Lozano-Quilis, H. Gil-Gomez, J. A. Gil-Gómez, S. Albiol-Perez, G. Palacios, H.M. Fardoum and A.S. Mashat, Virtual reality system for multiple sclerosis rehabilitation using KINECT, 2013 7th International Conference on Pervasive Computing Technologies for Healthcare (PervasiveHealth) (2013), 366-369.

[15] M. Murie-Fernandez and E. Ochoa, Virtual reality games combined with normal rehabilitation are an effective alternative in multiple sclerosis patients, 8th International Congress for NeuroRehabilitaton (2014).

[16] J. Finkelstein, O. Lapshin, H. Castro, E. Cha and P.G. Provance, Home-based physical telerehabilitation in patients with multiple sclerosis: A pilot study, J. Rehabil. Res. Dev. 45 (2008), 1361-1373.

[17] B. Freedman, A. Shpunt, M. Machline and Y. Arieli, U.S. Patent No. 20100118123 A1 (2010). 